\title{
Universidades Promotoras de la Salud: Reflexión para su implementación desde los determinantes sociales de la salud
}

\author{
Health Promoting Universities: A reflection for its implementation from the social determinants of health
}

Alba Yaneth Rincón-Méndez' orcid.org/0000-0003-3441-0430

Blanca Patricia Mantilla-Uribe ${ }^{*}$ orcid.org/0000-0002-9404-4367

1 Instituto Proinapsa, Universidad Industrial de Santander. Bucaramanga, Colombia

Fecha de recepción: Noviembre 28 - 2018

Fecha de revisión: Junio 12 - 2019 Fecha de aceptación: Diciembre 19 - 2019

Rincón-Méndez AY, Mantilla-Uribe BP. Universidades Promotoras de la Salud: Reflexión para su implementación desde los determinantes sociales de la salud. Univ. Salud. 2020;22(1):24-32.DOI: https://doi.org/10.22267/rus.202201.171

\section{Resumen}

Introducción: Los determinantes sociales de la salud (DSS) son las condiciones sociales y económicas que influyen en las diferencias individuales y colectivas en el estado de salud, las universidades pueden ser actores importantes en estos procesos. Objetivo: Identificar las concepciones de profesionales de Universidades Promotoras de la Salud (UPS) de Costa Rica, Puerto Rico, Perú, México y Colombia, sobre DSS, su relación y las formas de articulación con la estrategia de UPS. Materiales y métodos: Estudio cualitativo con enfoque descriptivo. Se desarrollaron 3 grupos focales con 35 personas. El análisis de la información se realizó según los parámetros de Miles et al. Resultados: Los participantes tienen una concepción clara de los DSS. Se identificaron como estrategias de intervención: formación del talento humano, implementación de espacios académicos en los currículos, inclusión del enfoque de habilidades psicosociales para la vida, sensibilización al personal directivo y docente. Conclusión: Es necesario que las universidades asuman un marco conceptual amplio sobre los DSS, para dirigir intervenciones y contribuir a la salud y la vida de las comunidades universitarias, aportar al debate y a la construcción de formas de acción política, económica y social orientadas a la eliminación de inequidades en salud.

Palabras clave: Universidades; determinantes sociales de la salud; promoción de la salud. (Fuente: DeCS, Bireme).

\begin{abstract}
Introduction: The social determinants of health (SDH) are the social and economic conditions that affect the individual and collective differences in the state of health. Universities can play an important role in these processes. Objective: To identify the conceptions that professionals from Health Promoting Universities (HPU) of Costa Rica, Puerto Rico, Peru, Mexico and Colombia have about SDH, their relationships, and the way to articulate them with the HPS strategy. Materials and methods: A qualitative study with a descriptive approach. 35 participants were divided into three focus groups. The analysis of the information followed the parameters proposed by Miels et al. Results: Participants have a clear conception of SDH. The following intervention strategies were identified: training of personnel, implementation of academic spaces in curriculums, inclusion of the approach of psychosocial skills for life, awareness of management and teaching staff. Conclusions: It is necessary that universities assume a broad conceptual framework on the social determinants of health. This aspect is important to (i) guide interventions and contribute to the health and life of university communities, (ii) contribute to the debate, and (iii) develop systems of political, economic and social action oriented towards the elimination of health inequities.
\end{abstract}

Keywords: Universities; social determinants of health; health promotion. (Source: DeCS, Bireme). 


\section{Introducción}

Los determinantes sociales de la salud (DSS) son un tema de especial interés para los diferentes organismos internacionales, como la Organización Mundial de Salud (OMS) que los define como las "condiciones en las que la gente nace, vive, trabaja y envejece" y establece diferencias en su trascendencia; se cataloga como de mayor importancia a los determinantes que generan una estratificación dentro de la sociedad, influyen en los puntos de vista de los individuos y establecen posiciones económicas dentro de las jerarquías de poder, prestigio y acceso a los recursos; ocasionando de esta forma inequidades en salud, que son injustas y evitables(1).

La Comisión mundial sobre DSS sugirió algunas estrategias prioritarias para la ejecución de medidas, enfatizando que "una buena salud exige la existencia de un sistema de salud de buena calidad, universal, integral, equitativo, eficaz, receptivo y accesible. Sin embargo, también depende de la participación de otros sectores y actores, y del diálogo con ellos, ya que sus actividades tienen un importante efecto sobre la salud"(2). En ese sentido, la universidad como escenario en donde circulan diversas personas con orígenes diferentes, con saberes y experiencias distintas y con capacidad para transformar su entorno cercano y distante, la convierten en un espacio propicio para dinamizar y abordar la salud desde el enfoque de determinantes sociales. Sin duda el rol de estas instituciones es muy importante teniendo en cuenta sus funciones principales: formación, investigación y extensión o vinculación con la comunidad.

En este contexto el concepto de Universidades Promotoras de la Salud (UPS) "hace referencia a las entidades de Educación Superior que fomentan una cultura organizacional orientada por los valores y los principios asociados al movimiento global de la Promoción de la Salud (PS), y se constata a través de una política institucional para la mejora continua y sostenida de las acciones de PS"(3). Un aspecto que se ha identificado como fundamental para el trabajo en UPS está relacionado con la intervención en los DSS, los cuales en el entorno universitario se vivencian de una forma específica, pues en este espacio coexisten grupos y jerarquías sociales (académicos, administrativos y estudiantes), con claros roles, que se interrelacionan por medio de complejos encuentros individuales y grupales, rodeados por factores estructurales de la institución, sus políticas y metas organizacionales. Adicionalmente, características individuales como estilos de trabajo, relaciones con los otros, percepción de control y autonomía y las características del refuerzo recibido por el trabajo/estudio realizado, afectan y modifican la percepción de bienestar en cada uno de quienes estudian o trabajan en una universidad(4).

Sin embargo, para avanzar en este campo es necesaria la discusión y el consenso con el fin de identificar opciones aplicables para la inclusión del enfoque de determinantes sociales de la salud en la promoción de la salud en el ámbito universitario. Teniendo en cuenta los anteriores planteamientos, se estableció como objetivo identificar las concepciones que las personas involucradas en las instituciones de educación superior tienen con relación a los DSS, así como las perspectivas para su intervención en el contexto universitario.

\section{Materiales y métodos}

Se realizó un estudio cualitativo con enfoque descriptivo (5). Este enfoque permite comprender los fenómenos, explorándolos desde la perspectiva de los participantes en un ambiente natural y en relación con su contexto, profundizando en sus puntos de vista, interpretaciones y significados(5). Dado que el enfoque de determinantes sociales tiene un fuerte sustento desde la PS, se invitó a participar a aquellas universidades que actúan desde este enfoque y hacen parte de la red de UPS pues cuentan con mayores claridades y elementos para aportar a la comprensión de los determinantes sociales y sus posibilidades de implementación. Las etapas llevadas a cabo en el proceso investigativo fueron:

Acercamiento a las personas vinculadas a la iniciativa de Universidades Promotoras de la Salud de instituciones educativas de Colombia, Costa Rica, Puerto Rico, Perú, México, quienes fueron contactadas a través de la Red Iberoamericana de Universidades Promotoras de la Salud-RIUPS. Para el caso de las personas de Colombia, se hizo a través de la Red Colombiana de Universidades e Instituciones de Educación Superior Promotoras de Salud REDCUPS. El desarrollo de los grupos focales se dio en el marco de un encuentro en Colombia sobre Universidades Promotoras de la Salud 
Se realizaron 3 grupos focales con 35 personas, pertenecientes a 14 universidades de cinco países, organizados de la siguiente manera: Dos grupos internacionales, cada uno de 10 personas, representantes de Costa Rica, Puerto Rico, Perú y México y otro grupo de 15 provenientes de Colombia. Las preguntas orientadoras utilizadas para el desarrollo de los grupos focales estuvieron dirigidas hacia:

- Concepciones sobre determinantes sociales de la salud.

- Importancia de los determinantes sociales de la salud en las Universidades Promotoras de la Salud.

- Posibilidades de implementación de la Estrategia de Universidades Promotoras de la Salud con el enfoque de Determinantes Sociales de la Salud.

La selección de los participantes fue intencional, utilizando el criterio de entrevistado especial, definido como aquellos que se ubican en una posición de observación y actuación única en la comunidad, en este caso específico en el contexto de las universidades promotoras de la salud(6). Las personas participantes están vinculadas a la Red de Universidades Promotoras de la Salud con el fin de aprender de las experiencias y hacer construcciones colectivas sobre las acciones a seguir en las Universidades. En la Tabla 1 se describen el perfil de las funciones de las personas participantes al interior de las universidades.

A cada participante se le explicó los objetivos de la investigación, la metodología empleada y los resultados esperados del estudio.

El análisis de la información obtenida siguió lo propuesto por Miles et al., quienes sugieren tres fases: obtener la información, capturar, transcribir y ordenar la información, codificar la información y finalmente integrarla(7). Durante el proceso de la investigación se tuvieron en cuenta los diferentes criterios de rigor de la investigación cualitativa: Las dos investigadoras con experiencia en investigación cualitativa participaron en el desarrollo de los grupos focales, cada grupo tuvo una duración de 2 horas. Una investigadora facilitó la conversación en los grupos focales, mientras la otra realizó observación, tomando notas clave para el análisis. Todas las trascripciones fueron leídas por duplicado, por cada una de las investigadoras, tomando nota de sus impresiones iniciales y posibles códigos. Posteriormente, los miembros del equipo revisaron y discutieron los tópicos, temas y citas de soporte, hasta obtener un consenso sobre las categorías emergentes para su análisis.

Tabla 1. Descripción del perfil de personas participantes

\begin{tabular}{lr}
\hline \multicolumn{1}{c}{ Funciones de los participantes } & $\mathbf{N}^{\circ}$ \\
\hline $\begin{array}{l}\text { Coordinación de programas de doctorado en salud } \\
\text { pública/doctorado en psicología. }\end{array}$ & 2 \\
$\begin{array}{l}\text { Coordinación de programas de pregrado en salud y } \\
\text { afines. }\end{array}$ & 2 \\
$\begin{array}{l}\text { Coordinación de comisión de participación de } \\
\text { estudiantes en la RedCups }\end{array}$ & 1 \\
$\begin{array}{l}\text { Coordinación de transverzalización de promoción de } \\
\text { la salud }\end{array}$ & 1 \\
$\begin{array}{l}\text { Coordinación de calidad y acreditación } \\
\text { Directores de servicios de bienestar universitario } \\
\text { Investigación en ciencias de la salud y promoción de } \\
\text { la salud }\end{array}$ & 2 \\
$\begin{array}{l}\text { Coordinación de programas de estilos de vida } \\
\text { saludables/ hábitos culturales y de salud. }\end{array}$ & 3 \\
$\begin{array}{l}\text { Profesionales de bienestar universitario } \\
\text { Docentes de cátedra en carreras como enfermería, } \\
\text { psicología, estomatología, medicina y educación. }\end{array}$ & 4 \\
\hline
\end{tabular}

\section{Consideraciones éticas}

Se solicitó a los participante consentimiento informado para la realización y grabación de los grupos focales, garantizando en todo momento el manejo ético, anónimo y confidencial de la información y del nombre de los participantes, respetando las normas de investigación en sujetos humanos estipuladas por la Declaración de Helsinki II y la Resolución 008430 de 1993 del Ministerio de Salud de Colombia por la cual se establecen las normas científicas, técnicas y administrativas para la investigación en salud. Teniendo en cuenta que se trata de una investigación de riesgo mínimo, se recibió aval del Consejo Directivo de Proinapsa-UIS según consta en el acta de № 29 de 13 de diciembre de 2017.

\section{Resultados}

A continuación, se describen los hallazgos de la investigación los cuales se presentan de manera integrada de las diferentes universidades y países participantes. 


\section{Concepciones sobre los determinantes sociales de la salud}

Los participantes manifestaron diversas maneras de entender los DSS; sin embargo, estas se pueden agrupar en dos: por un lado aquellas concepciones que están enfocadas a la transformación y modificación ya sea de aspectos individuales Determinantes entendidos como la modificación de conductas -o contextuales -Determinantes entendidos como la modificación de contexto político económico y sociocultural y por otro las enfocadas a entender los Determinantes como aquellos aspectos que condicionan la situación de salud de las personasDeterminantes entendidos como factores que definen el estar o no saludable-.

Al reflexionar sobre los DSS como instrumento para la modificación de las conductas, se evidencia en el discurso de los participantes la comprensión de la salud más allá de la decisión individual y por lo tanto más allá de las conductas y los estilos de vida que se asuman de manera individual; pues dan un peso importante al contexto político, económico y social para su transformación, así como la necesidad de actuar sobre estos aspectos: "Condiciones culturales, políticas y financieras que actúan sobre las conductas"[Participante, México]. "Aquellos que transforman los aspectos culturales, políticos $y$ financieros, los cuales a su vez influyen sobre las conductas en salud" [Participante, Costa Rica]

También los determinantes sociales de la salud fueron relacionados con la modificación del contexto político, económico y sociocultural. Se destaca la necesidad de actuar sobre aspectos que influyen en la salud: "Implican servicios, estados, gobiernos, políticas; orientadas a la promoción de la salud, condiciones de vida digna y equidad y a la intervención de los factores asociados a las desigualdades sociales" [Participante, Perú]. "Modificar elementos del medio o contexto que afectan al individuo, la comunidad y la familia actuando en forma positiva o negativa" [Participante, Colombia].

Así mismo, otros plantearon una concepción de los determinantes como aquellos aspectos que afectan el estar o no saludable, sin mencionar que los determinantes sociales de la salud no solo condicionan la situación de salud de las personas, sino la forma en que esta se distribuye y se produce de acuerdo a diferencias que son de orden estructural como las políticas, la ocupación, el nivel social, el género y la etnia, entre otros, es decir las inequidades sociales de la salud:"Condiciones sistémicas estructurales que definen la posibilidad de estar o no estar saludable" [Participante, Puerto Rico]. "Condiciones con capacidad para impactar positivamente la salud a nivel individual, social, del ambiente" [Participante, Colombia]. "Factores que impactan en la salud en general" [Participante, Perú].

\section{Determinantes sociales de la salud en el quehacer de las universidades promotoras de la salud}

Existe un acuerdo respecto al abordaje de los DSS, teniendo en cuenta la lógica de las funciones sustantivas de las instituciones de educación superior: formación, investigación y extensión a la comunidad.

\section{Formación del talento humano, una vía para la transformación}

Se presenta consenso en relación a la importancia de la inclusión de los DSS en el plan de estudios, la formación de directivos y docentes y el empoderamiento de la comunidad universitaria, como mecanismos que permiten realizar en el mediano y largo plazo transformaciones no solamente individuales sino colectivas en las personas de la comunidad académica.

En cuanto a la inclusión de los DSS en el plan de estudios, las personas entrevistadas identifican como aspectos clave la comprensión de la salud desde un enfoque integral, la inclusión en diversas asignaturas, la enseñanza de las habilidades psicosociales para la vida y el énfasis en la práctica de la promoción de la salud. Para ellas, hacer realidad este enfoque implica comprender la salud como un asunto que va más allá de la ausencia de la enfermedad y que por tanto trasciende la mirada biomédica o medicalizada, que es un asunto transversal a las disciplinas y que por tanto requiere de miradas integrales y holísticas. "En la medida en que se amplíe el concepto, serán más las facultades y escuelas universitarias que incluyan este concepto y sus determinantes en el análisis crítico de su quehacer profesional" [Participante, Costa Rica].

Lo anterior, permitirá hacer una valoración diferente de la salud y vincular a otros actores diferentes al sector salud como corresponsables de la salud individual y de los colectivos humanos. 
Así mismo, se hace necesario realizar transformaciones en los planes de estudio, incluir cursos o seminarios específicos tanto en pregrado como posgrado, entre otros. "Transformar el plan de estudios de todas las facultades, pues la salud es de todos y los determinantes deberían conocerlos todos nuestros estudiantes" [Participante, México]. "Como componentes transversales en las carreras de pregrado, el reto mayor se centra en que en carreras distintas al área de la salud se incluya la salud desde un enfoque integral, se pueda revisar la corresponsabilidad de todos los campos disciplinares en cuanto a la promoción de la salud y los determinantes sociales" [Participante, Colombia].

La enseñanza y el aprendizaje de las habilidades psicosociales para la vida, surgen en el discurso de las personas participantes como un elemento clave que puede ser abordado por las universidades, el cual puede incidir de manera directa en la consecución de estilos de vida saludables; cabe mencionar que esta mirada ya está siendo implementada por algunas instituciones a través de programas de bienestar universitario. "De hecho trabajamos ya en habilidades para la vida, para la toma de decisiones en lo que tiene que ver con los estilos de vida. Los programas de bienestar tienen ese enfoque, estilos de vida como decisión responsable". [Participante, Colombia].

Pese a lo anterior, el hecho de que los programas surgen de los responsables de los servicios de salud de las universidades, se identifica como un factor que dificulta la vinculación con la parte académica que es finalmente la que realiza mayores reflexiones sobre la salud. Los participantes consideraron fundamental tener en cuenta el contexto de la juventud que asiste a las instituciones de educación superior, pues se encuentran enfrentados a múltiples situaciones familiares, económicas, lejos de sus casas, entre otros, lo que hace aún más pertinente el fortalecimiento de las habilidades psicosociales para la vida.

Teniendo en cuenta las concepciones de determinantes sociales de la salud en relación a la transformación del contexto, manifestadas por el grupo de participantes, se identifica la necesidad de la formación de personas críticas, empoderadas, con capacidad para modificar su contexto y participar activamente en las decisiones políticas que afectan la vida de las poblaciones. "El currículo debe ir más allá de lo disciplinar y apoyar el desarrollo de habilidades para la vida, para que los estudiantes transformen su propio entorno" [Participante, Colombia]. "Necesitamos líderes y políticos que entiendan de forma diferente la salud, sensibles a las realidades sociales" [Participante, Colombia]. "Las universidades deben tener líderes capaces de participar en el trabajo interinstitucional, local, regional y nacional" [Participante, Perú].

Por su parte, la formación de las personas directivas y docentes de las instituciones educativas surge como un aspecto clave para viabilizar los procesos, la asignación de recursos y finalmente lograr las transformaciones sociales y culturales deseadas en el marco de las universidades promotoras de la salud. En esta medida identifican un aspecto clave el desarrollo de acciones de cabildeo que permitan llevar esta estrategia a los tomadores de decisión del contexto universitario. "Es necesario formar a directivos y docentes de todas las facultades o escuelas universitarias por la responsabilidad que se tiene como universidad promotora de la salud, el discurso debe ser políticamente atractivo para los directivos con el fin que además que den vía libre al proceso, asignen recursos para ello". [Participante, Colombia].

Lo anterior permite, desde la perspectiva de los participantes, transcender la dinámica de isla en la que se mueven algunas instituciones "Las universidades no se mueven y actúan como un todo, lo cual impide el desarrollo de políticas y de acciones integrales en torno a la promoción de la salud y los determinantes sociales de la salud" [Participante, Puerto Rico]."El trabajo aislado de las facultades, impide la formación de profesionales con un enfoque de cuidado de la salud, identificación e intervención de los determinantes sociales" [Participante, Colombia].

Finalmente, los participantes hacen una reflexión en cuanto a la promoción de la salud como una herramienta para el abordaje de los determinantes sociales de la salud. "Comprender mejor la promoción de la salud desde la práctica contribuirá seguramente al desarrollo de mayores capacidades para incluir el enfoque de determinantes sociales de la salud en la universidad" [Participante, Costa Rica].

\section{Extensión Universitaria: Una herramienta clave para las Universidades Promotoras de la Salud}

Se reconoce que las universidades responden a necesidades específicas de su contexto, y es en esta 
medida que la extensión se valora como una actividad que hace énfasis en la relación de la institución con la comunidad en la que está inmersa, estableciendo un diálogo para la transformación de las realidades mediante la aplicación del conocimiento, metodologías y aprendizaje del estudiantado. Bajo esta lógica y reconociendo las concepciones de los determinantes sociales de la salud, las personas manifiestan la necesidad de: "Hacer análisis de la comunidad desde el enfoque de determinantes sociales, para que la universidad como un todo pueda intervenir en los diferentes determinantes de orden estructural e intermedio" [Participante, México].

Lo anterior se puede lograr siempre y cuando se establezca una relación horizontal, situada, dialógica entre la institución y la comunidad para de esta manera acercarse a la comprensión de las realidades, identificar formas de trabajo colaborativo, lograr su participación en los procesos de identificación de problemas, soluciones y seguimiento: "Conocer a las comunidades con las cuales se trabaja, analizando su salud y los determinantes sociales que la caracterizan" [Participante, Perú]. "Identificar las inequidades apoyados en la participación, buscar soluciones con trabajo interdisciplinario y participación de la comunidad, los docentes y los estudiantes" [Participante, México].

Pese a lo anterior, también se reconoce que en algunas ocasiones las universidades trabajan con las comunidades basadas en un sistema de oferta y no de análisis de sus necesidades y de las formas de contribuir a su solución. Una estrategia para aunar esfuerzos y potencializar las acciones, tiene que ver con la generación de una agenda en las universidades y una articulación directa con los escenarios gubernamentales desde sus diferentes niveles para la gestión de determinantes sociales de la salud, que le permitan apoyar acciones y orientar a los tomadores de decisiones. Se valora el carácter académico y científico de las universidades como una vía para la vinculación de los saberes que allí se generan en las discusiones políticas. Asimismo, las universidades cuentan con reconocimiento público de su saber, lo cual es un factor importante que les permite incursionar y hacer aportes sobre formas efectivas de analizar la salud desde los determinantes sociales de la salud. "Es posible generar un plan de acción inter-universidades que integre a todos los actores: entes territoriales, comunidad, sector salud, ONG en un marco político con seguimiento $y$ trabajo intersectorial" [Participante, Colombia].

\section{Determinantes sociales de la salud e investigación en la universidad}

Las personas que participaron expresaron la importancia de la investigación en el marco de los determinantes sociales, en particular plantearon que la investigación con este enfoque es necesaria para analizar los problemas y reconocer las inequidades. Así mismo, aceptan que la puesta en marcha de investigaciones bajo esta lógica requiere del fortalecimiento del enfoque cualitativo y la mejora de las prácticas pedagógicas.

Respecto al fortalecimiento del enfoque cualitativo expresaron que este actúa como una herramienta que facilita una mejor comprensión de la salud en los contextos sociales, en esa medida permite conocer la realidad de la comunidad educativa y reconocer los diferentes grupos sociales, sus oportunidades, dificultades y desventajas. "Preguntarse cómo viven, estudian y trabajan quienes hacen parte de la comunidad educativa". [Participante, Perú].

Igualmente, destacaron la importancia de las líneas de base y la realización de mapeo de activos que permitan a las universidades conocer sus propias fortalezas $y$ debilidades como instituciones promotoras de la salud en el marco de determinantes sociales. En particular, la sistematización como metodología de investigación cualitativa fue citada por los participantes como una manera de recoger y difundir experiencias exitosas sobre inclusión de determinantes sociales de la salud, desde el enfoque de promoción de la salud, las cuales se conviertan en una oportunidad de aprendizaje, de visibilización de beneficios. “Construcción y recopilación de evidencias en torno a este campo de la salud y al fortalecimiento del movimiento de universidades promotoras de la salud" [Participante, Colombia].

En cuanto a la mejora de las prácticas pedagógicas se hizo alusión a la investigación como herramienta para mejorar prácticas pedagógicas, sujeta a la necesidad de que la universidad realice cambios internos que le permitan actuar en concordancia con el movimiento de universidades promotoras de la salud y con el enfoque de determinantes sociales de la salud. En ese sentido, se hace necesaria la revisión 
de la pertinencia del plan de estudios en cuanto a investigación, especialmente en los seminarios de investigación y su relación con la formación de profesionales críticos con capacidad para transformar contextos desde las distintas disciplinas.

\section{Discusión}

La universidad es reconocida como escenario propicio para dinamizar y abordar la salud desde el enfoque de determinantes sociales, pues tiene un rol protagónico en las transformaciones sociales y un imperativo de ponerse en consonancia con las necesidades sociales, económicas, políticas y ambientales de su propio contexto(8). En el ámbito internacional tanto la Organización Panamericana de la Salud (OPS) como la OMS han reconocido su relevancia al señalar que estas instituciones promueven y apoyan la colaboración entre los sectores del gobierno, los sectores privado y público y los socios no gubernamentales de cada país; por su parte desde el movimiento de UPS se ha demostrado que es una iniciativa idónea para fortalecer la salud mediante el ideario y la praxis de la promoción de la salud(9). Así mismo, las universidades son instituciones peculiares debido, entre otras cosas a que son centros de enseñanza con papeles de formación, educación en investigación, son centros de creatividad e innovación, en los que se aplica el conocimiento desde enfoques disciplinares e interdisciplinares ${ }^{(10)}$.

Los hallazgos de este estudio permiten identificar la inclusión del enfoque de los determinantes sociales de la salud en el ámbito universitario como un factor de vital importancia para hacer realidad la estrategia de universidades promotoras de la salud. Al revisar la literatura no se encuentra información muy amplia sobre cómo hacer la inclusión de determinantes sociales de la salud en el ámbito universitario, lo cual indica la necesidad de realizar este tipo de estudios que aporten a la construcción de nuevo conocimiento. Las dimensiones identificadas por las personas participantes para la operativización de la estrategia están relacionadas con las funciones sustantivas de las instituciones de educación superior: la formación, la investigación y la extensión.

En cuanto a la formación del talento humano, este estudio devela la importancia de trabajar desde diferentes flancos: la inclusión de contenidos en las mallas curriculares, la generación de cursos y seminarios en pregrado y posgrado, la sensibilización del personal administrativo $\mathrm{y}$ docente. Estos aspectos han sido reconocidos por otros estudios en los cuales se menciona la necesidad de incorporar ejes temáticos de promoción de la salud y determinantes sociales de la salud en las mallas curriculares de todas las carreras de pre-grado e impulsar cursos de post grado, diplomados y maestrías relacionados (11).

Un aspecto identificado en el estudio tiene que ver con la necesidad de incluir la formación en habilidades psicosociales para la vida como una vía para contribuir en la consecución de estilos de vida saludables. Esta mirada desde las habilidades es un aspecto aún incipiente en otros contextos; sin embargo, la perspectiva hacia los estilos de vida es reiterativa con lo reportado en otros estudios en los cuales se identifica que las instituciones de educación superior deben, entonces, empoderarse en su responsabilidad como promotoras de la salud comenzando por el apoyo cotidiano de cada uno de sus miembros y la generación de entornos que promuevan estilos de vida cada vez más saludables(12). En esa medida también se identifican retos relacionados con la necesidad de concentrar y aunar esfuerzos en la promoción de estilos de vida saludable con la participación de todos los involucrados y el compromiso desde los entes correspondientes, con la creación de entornos que promuevan la salud y el bienestar de la comunidad universitaria, trabajando a la vez, sobre las conductas de los individuos, con el fin de alcanzar los objetivos propuestos y que lo logrado se mantenga a través del tiempo(13).

Tal y como reporta Santos, el comportamiento de las personas a lo largo de su vida, frente a distintos riesgos para su salud, es uno de los factores determinantes más estudiado desde la segunda mitad del siglo XX(14). Expresa, además, que algunos estilos de vida pueden afectar la salud de las personas y que estos, se escogen libremente. Sin embargo, actualmente se sabe que, en la adopción de estilos de vida, no sólo influye la decisión personal sino otros aspectos de índole político, social y económico que deben tenerse en cuenta a fin de no ligar las condiciones de salud a la responsabilidad exclusiva de los individuos. Los estilos de vida colectivos no obedecen a decisiones personales sino a conductas influenciadas por las oportunidades 
definidas por el medio social en que las personas viven(15), lo cual está en relación directa con los DSS.

Un elemento emergente del presente estudio tiene que ver con el rol de las directivas de las instituciones educativas en la puesta en marcha de las iniciativas y la sostenibilidad de las mismas, estos aspectos ya han sido reportados por otros estudios en los cuales se reconoce que las dinámicas administrativas, la estructura, políticas y decisiones estratégicas que se adopten desde los cargos directivos de las universidades tienen un impacto directo. El desarrollo de políticas institucionales es fundamental para la creación y mantenimiento de programas, dando sustento económico y facilitando el desarrollo de las actividades(16). La voluntad política institucional y de los actores comprometidos es un factor clave de éxito para garantizar su continuidad(17).

En cuanto al rol de la extensión universitaria, las personas participantes reconocen la importancia de desarrollar las acciones de acercamiento a la sociedad en el marco de los determinantes sociales de la salud, por su poder explicativo, comprensivo y transformador. El papel de la extensión universitaria ha sido reconocido en diversos estudios en los cuales se ve como una importante función social y un proceso formativo integrador de los vínculos Universidad-Sociedad; su desarrollo es vital en la formación de una sociedad preparada para asimilar los diferentes escenarios, contribuyendo a transformarla(18); su práctica permite que la universidad conozca las tecnológicas y formas de abordaje de la salud que se producen en la comunidad(19); posibilita un dialogo permanente entre la universidad con el fin de generar bienestar(20); es un aporte a la construcción de capital social al facilitar la construcción de redes basadas en la confianza(21); permite validar el uso social del conocimiento y reafirmar los saberes propios de la universidad como pertinentes en un contexto determinado(22).

Teniendo en cuenta el método utilizado para el desarrollo de la investigación se seleccionaron personas que estuvieran vinculadas a UPS, pero que no necesariamente tuvieran conocimiento sobre DSS. Sin embargo, se reconoce como limitación de la misma, que si bien estos resultados identifican acuerdos con relación a los determinantes sociales de la salud y su implementación en el ámbito de las
UPS, estos se circunscriben al contexto de las personas participantes, por tanto no puede ser generalizada a otras poblaciones o escenarios distintos.

Esta investigación abre el debate entre quienes han trabajado en UPS, sobre cómo articular los determinantes sociales de la salud, sus resultados son un insumo importante para continuar interactuando con otras universidades que desarrollan la estrategia. Sin embargo, será necesario realizar otras investigaciones en donde se vincule a universidades que no han tenido la experiencia de ser promotoras de la salud, con el fin de establecer comparaciones respecto a la comprensión de los determinantes sociales de la salud.

\section{Conclusiones}

Siendo las universidades un escenario para la libre discusión, entidades que son escuchadas y tenidas en cuenta en escenarios políticos y decisorios de los países, resulta conveniente que especialmente las universidades promotoras de la salud asuman un marco conceptual más amplio con relación a la promoción de la salud y a los determinantes sociales de la salud, que oriente y facilite las intervenciones y permita además de contribuir internamente a la salud y la vida de las comunidades universitarias a través del debate a la construcción de formas de acción política, económica y social orientadas a la eliminación de las inequidades.

El rol de las universidades debe ir más allá de definir los determinantes sociales como posibles causas que afectan la salud de las personas, e identificar grupos a intervenir según etnia, lugar de origen o ingresos pues el abordaje de los mismos implica transformar el contexto y las inequidades en salud.

En la medida en que las universidades apropien desde sus áreas misionales el enfoque de determinantes sociales, podrá contribuir al desarrollo de capacidades de los nuevos profesionales en diferentes campos de desarrollo. Esta investigación contribuye al debate sobre los retos que tienen las universidades en este campo y enriquece la discusión sobre las formas en que se pueden articular los determinantes sociales a su quehacer institucional. 
Conflicto de intereses: Ninguno a declarar.

\section{Referencias}

1. Organización Mundial de la Salud, Comisión sobre Determinantes sociales de la salud. Subsanar las desigualdades en una generación: Alcanzar la equidad sanitaria actuando sobre los determinantes sociales de la salud[Internet]. Ginebra: OMS; 2009. [citado 20 de agosto de 2018]. Disponible en: http://www.who.int/social_determinants/thecommission/f inalreport/es/

2. Organización Mundial de la Salud. Declaración política de Río sobre determinantes sociales de la salud[Internet]. Río de Janeiro: OMS; 2011. [citado 20 de agosto de 2018]. Disponible en: http://www.who.int/sdhconference/declaration/Rio_politi cal_declaration_Spanish.pdf

3. OPS/OMS. Acerca de las Universidades Promotoras de Salud[Internet].Washington: OPS/OMS; 2015.[citado 20 de agosto de 2018]. Disponible en: https://www.paho.org/hq/index.php?option=com_content \&view=article\&id=10669:2015-about-health-promotinguniversities\&Itemid $=820$ \&lang $=\mathrm{es}$

4. Dávila AL, Rodríguez MC, Pérez D. El índice de desarrollo humano; instrumental para las universidades promotoras de la salud [Internet]. Alicante: Universidad de Alicante, Proyecto Universidad Saludable; 2017. [citado 28 de noviembre de 2018]. Disponible en: http://rua.ua.es/dspace/handle/10045/67351

5. Ulin PR, Robinson ET, Tolley EE. Investigación aplicada en salud pública métodos cualitativos. Washington: OPS; 2006.

6. Ventura-León JL, Barboza-Palomino M. El tamaño de la muestra: ¿Cuántos participantes son necesarios en estudios cualitativos? .Rev Cuba Inf En Cienc Salud. 2017;28(3):1-2. Disponible en: http://scielo.sld.cu/scielo.php?script=sci_arttext\&pid=S230 7-21132017000300009

7. Miles MB, Huberman AM, Huberman MA, Huberman PM. Qualitative Data Analysis: AnExpandedSourcebook. SAGE; 1994. 338.

8. Beltrán-Llevador J, Íñigo-Bajo E, Mata-Segreda A. La responsabilidad social universitaria, el reto de su construcción permanente. RevIberoamEducSuper. 2014; 5(14):3-18. Disponible en: http://www.scielo.org.mx/scielo.php?script=sci_arttext\&pi $\mathrm{d}=\mathrm{S} 2007-28722014000300001$

9. Arroyo-Acevedo H, Durán Landazabal G, Gallardo Pino C. Diez años del Movimiento de Universidades Promotoras de la Salud en Iberoamérica y la contribución de la Red Iberoamericana de Universidades Promotoras de la Salud (RIUPS). GlobHealthPromot. 2015;22(4):64-8. Disponible en:

https://journals.sagepub.com/doi/abs/10.1177/17579759 14547548?journalCode $=$ pedb

10. Lara N, Saldaña Y, Fernández N, Delgadillo HJ. Salud, calidad de vida y entorno universitario en estudiantes mexicanos de una universidad pública. Hacia Promoc Salud. 2015;20(2):102-17. Disponible en: http://www.scielo.org.co/pdf/hpsal/v20n2/v20n2a08.pdf
11. Heraud SB. Universidades saludables: Una apuesta a una formación integral del estudiante. RevPsicol. 2013;31(2):287-314. Disponible en: http://www.scielo.org.pe/pdf/psico/v31n2/a06v31n2.pdf

12. Fabelo Roché JR, Iglesias Moré S, Gómez García AM. La promoción de salud en la Universidad de Ciencias Médicas de La Habana. Rev Habanera Cienc Médicas. 2017;16(1):93105. Disponible: http://scielo.sld.cu/scielo.php?script=sci_arttext\&pid=S172 9-519X2017000100010

13. Coronel-Carbo J, Marzo-Páez N. Determinantes sociales y de salud protectores en el ámbito universitario. MEDISAN. 2018;22(8):825-37. Disponible en: http://scielo.sld.cu/pdf/san/v22n8/1029-3019-san-22-08825.pdf

14. Santos Padrón H. Los determinantes sociales, las desigualdades en salud y las políticas, como temas de investigación. Rev Cuba Salud Pública. 2011;37(2):136-144. Disponible http://scielo.sld.cu/scielo.php?script=sci_arttext\&pid=S086 4-34662011000200007

15. Pupo-Ávila NL, Hernández-Gómez LC, Presno-Labrador C. La formación médica en Promoción de Salud desde el enfoque de los determinantes sociales. Rev Cuba Med Gen Integral. 2017;33(2):218-29. Disponible en: http://scielo.sld.cu/scielo.php?script=sci_arttext\&pid=S086 4-21252017000200007

16. Bravo-Valenzuela $\mathrm{P}$, Cabieses B, Zuzulich MS, Muñoz M. Glosario para universidades promotoras de la salud. RevSalud Pública. 2013;13. Disponible en: http://www.scielo.org.co/pdf/rsap/v15n3/v15n3a15.pdf

17. Gaviria-Méndez AE. La Universidad de Antioquia como institución promotora de la salud (Medellín, Colombia, 2010-2013). Investig En Enferm Imagen Desarro. 2016;18(1):13-30. Disponible en: https://revistas.javeriana.edu.co/index.php/imagenydesarr ollo/article/view/10687

18. López M. Extensión universitaria y salud pública en Argentina como caso testigo en la región | Revista de Investigación Educativa. RevInvestigEduc. 2016;34(1). Disponible en: http://revistas.um.es/rie/article/view/215411

19. Leitão, C. Extensión universitaria brasileña: arte, cultura, ciencia y tecnología en favor del desarrollo humano. Agenda Cultural Alma Máter. 2018.

20. Martí J, Calderón A, Fernández A. La responsabilidad social universitaria en Iberoamérica: análisis de las legislaciones de Brasil, España y Perú. Revista iberoamericana de educación superior. 2018; 9(24)107-124. Disponible en: http://www.scielo.org.mx/scielo.php?script=sci_abstract\&p id=S2007-28722018000100107\&lng=es\&nrm=iso

21. Williamson G, Contreras N. Universidad en el siglo XXI: ¿extensión, responsabilidad social universitaria, vínculo con el medio o conocimiento para el desarrollo de los territorios?. Revista Espaço Pedagógico. 2018;25(3): 602623.

22. González Fernández M, González R. Retos de la extensión en la universidad innovadora. Congreso Universidad. 2018; $7(2): 1-11$ 